\title{
Identification and heterologous expression of a Sarcoptes scabiei cDNA encoding a structural antigen with immunodiagnostic potential
}

\author{
Rosa CASAIS ${ }^{\mathrm{a}}$, Miguel PrIETO ${ }^{\mathrm{a}}$, Ana BALSEIRO ${ }^{\mathrm{a}}$, Paloma SOlANO ${ }^{\mathrm{a}}$, \\ Francisco PARRA ${ }^{\mathrm{b}}$, José M. MARTín ALONSO ${ }^{\mathrm{b} *}$ \\ ${ }^{a}$ Servicio Regional de Investigación y Desarrollo Agroalimentario (SERIDA), \\ Laboratorio de Sanidad Animal, Jove, Gijón, Asturias, Spain \\ ${ }^{\text {b }}$ Departamento de Bioquímica y Biología Molecular, Instituto Universitario de Biotecnología \\ de Asturias, Universidad de Oviedo, Edificio Santiago Gascón, Campus El Cristo, 33006 Oviedo, Spain
}

(Received 21 July 2006; accepted 20 December 2006)

\begin{abstract}
The mite Sarcoptes scabiei causes sarcoptic mange (or scabies), a disease of considerable human and veterinary significance. An S. scabiei cDNA clone of about $2 \mathrm{~kb}$ was isolated from a $S$. scabiei var. hominis expression library by immunological screening using blood serum from a naturally infected chamois (Rupicapra rupicapra). The nucleotide sequence of the identified cDNA contains an open reading frame of $1930 \mathrm{bp}$ that encodes a 642 amino acid polypeptide. This polypeptide shows tandem repeats of a glycine-serine rich 20 residue sequence followed by a unique C-terminal glutamate rich 54 residue sequence. The cDNA or the deduced polypeptide did not show significant similarities to any of the sequences in the databases. A carboxyl-terminal fragment of this polypeptide (residues 380 to 642) was efficiently expressed in Escherichia coli as a fusion with Glutathione S-transferase and then was used to produce a specific antiserum. The antigen encoded by the cDNA was located at the integument of the mite's epidermis and the cavities surrounding its vital organs. Western blot analysis of mite extracts using the specific antiserum against the recombinant protein identified antigens larger that $60 \mathrm{kDa}$ indicating that the isolated cDNA did not contain the full ORF. Moreover, we designed a diagnostic assay based on the carboxyl-terminal fragment of the antigen for the identification of infected animals.
\end{abstract}

Sarcoptes scabiei / cDNA library / immunolocalisation / sarcoptic mange diagnosis / ELISA

\section{INTRODUCTION}

Scabies or sarcoptic mange is a disease of global distribution affecting both human and animal populations.

The disease results from Sarcoptes scabiei skin infection. The sarcoptes mites tunnel into the skin where their feeding and laying of eggs causes the introduc-

*Corresponding author: jmmartin@uniovi.es tion of many antigenic proteins into the host system causing irritation and inflammation, leading to a host immune response and antibody production. This host response combined with the damage caused by the sarcoptes mites and provocation of severe scratching lead to the formation of skin cracking, hyperkeratinisation and loss of hair.

In Europe, several ungulate populations have suffered epizootics of scabies. Severe 
outbreaks of sarcoptic mange in chamois have been described in Europe, including the eastern Alps and Cantabrian mountains of northwestern Spain [9, 32]. Currently outbreaks of the disease, affecting the populations of chamois and red deer (Cervus elaphus), are in progress over a significant part of their distribution area in the northwest of Spain. Hence, resource managers have demanded improved methods to diagnose the disease and fight against its spread.

The correct diagnosis of scabies is not easy to establish using conventional monitoring tools such as the observation of skin lesions or behaviour, especially in the case of wild animals. In infected swine herds most of the animals are subclinically infected, so that the skin lesions are usually not visible or not very specific $[7,19]$. A conclusive diagnosis of scabies is only possible by microscopic observation of the mite in skin scrapings. This method is $100 \%$ specific but frequently has a low sensitivity since the number of parasites present in the samples is often very low. It has been reported that more than $50 \%$ of skin scrapes can be negative in canine scabies infections [24]. In recent years, many enzyme-linked immunosorbent assays (ELISA), based on crude mite extracts have been developed for several host species $[6,7,13,31,36]$. $S$. scabiei is the source of multiple antigenic molecules [1-3] and the varieties of $S$. scabiei from different animals introduce both cross-reactive and variety specific antigens into the host $[5,13]$. Consequently, antigens in extracts made from one variety of scabies mite can be used to detect serum antibodies elicited by another variety $[7,13,23]$.

Problems derived from the use of antigen preparations obtained from whole mites such as the complexity of total homogenates and the difficulty in obtaining sufficient quantities of the parasite from dead or live infected animals, could be overcome using pure recombinant antigens expressed in Escherichia coli or other heterologous hosts. This approach has been successfully used previously for the expression of other parasite antigens [15, 18, 20,30].

The aim of this present study was to identify relevant antigens of S. scabiei that could be used to develop a reliable, sensitive and specific mange diagnostic test for the identification of infected animals.

\section{MATERIALS AND METHODS}

\subsection{Parasites}

S. scabiei mites of both sexes and different developmental stages were obtained from skin samples of local infected wild animals. Each skin sample was incubated in a glass Petri dish at $37{ }^{\circ} \mathrm{C}$ for $24 \mathrm{~h}$. Afterwards, the mites were observed using magnification lenses (Olympus SZX9, Tokyo, Japan), collected from the surface of the dish in $70 \%$ ethanol and pelleted by centrifugation at $300 \times g$ for $10 \mathrm{~min}$ at room temperature. The pellet was suspended in $1 \mathrm{~mL}$ PBS and homogenised with 60 strokes in a glass Dounce homogeniser (Bellco Glass, Inc., Vineland, NJ, USA). The lysate was transferred to a $1.5 \mathrm{~mL}$ microcentrifuge tube and subjected to centrifugation at $900 \times g$ for $5 \mathrm{~min}$ at room temperature. The supernatant was concentrated to $0.37 \mu \mathrm{g} / \mu \mathrm{L}$ by lyophilisation and was kept at $-20{ }^{\circ} \mathrm{C}$ until further use.

\subsection{Bacteria}

Escherichia coli XL1-Blue (Stratagene, La Jolla, CA, USA) were used in routine plasmid construction. E. coli BL21 were used for production of the GST$\lambda 20 \Delta \mathrm{B} 3$ fusion protein. The strains XL1Blue MRF' and XLOLR (Stratagene) were 
used as hosts for the bacteriophage $\lambda$ ( $\lambda$ ZAP express, Stratagene). XLOLR are designed to allow efficient in vivo excision of the $\mathrm{pBK}-\mathrm{CMV}$ phagemid vector from the ZAP Express vector while preventing problems associated with helper phage coinfection.

\subsection{Recombinant DNA techniques}

The standard procedures for recombinant DNA manipulation were carried out according to the manufacturers' instructions or following the methods described in laboratory manuals [33].

\subsection{Immunological library screening}

The $S$. scabiei amplified cDNA library Yv4 used in this study was kindly provided by Dr David J. Kemp (Malaria and Scabies Laboratory, Queensland Institute of Medical Research, Brisbane, Australia) and contained an average insert size of $1.6 \mathrm{~kb}$ [10] flanked by EcoRI and XhoI restriction sites in the vector $\lambda$ ZAP express (Stratagene). Nitrocellulose filters containing $\lambda$ ZAP clones from the amplified library were screened with a 1:20 dilution of a serum taken from a naturally infected chamois (this serum was also used as a positive reference in ELISA tests) using a previously described procedure [33]. The immunocomplexes were detected using a 1:1500 dilution of protein $\mathrm{G}$ peroxidaseconjugated (Sigma, St. Louis, MO, USA) and 4-chloro-1-naphthol as chromogenic substrate (Sigma). Positive plaques were re-screened at a lower density until plaque pure populations were obtained. This process was followed by in vivo excision of pBK-CMV phagemids from the $\lambda$ ZAP express vectors following the instructions of the manufacturer.

\subsection{Sequence analysis}

High titre phage clones were excised in vivo as pBK-CMV phagemids with the
ExAssist helper phage and sequenced using the T7 and T3 promoter primers. Sequence analyses of plasmid DNA (recombinant pBK-CMV phagemids and pGEX constructs) were done with a BigDye(r) Terminator v3.1 kit. Sequence reactions were analysed on an ABI PRISM 3100 Genetic Analyser. The nucleotide and deduced amino acid sequences were analysed with the aid of the DM5 program [25]. The hydropathicity plot was calculated according to Kyte and Doolittle [16]. For sequence similarity and homology searching, the Fasta program [29] at the European Bioinformatics web server using the default parameters and also the Blast program at the NCBI web server were used.

\subsection{Construction of pGEX-4T3-Ss $\lambda 20$ and derivatives}

In order to produce the selected $S$. scabiei specific antigen as a fusion protein, the positive clone Ss $\lambda 20$ was excised as a phagemid that was double-digested with the restriction endonucleases EcoRI and XhoI. The restriction fragment corresponding to the $S$. scabiei cDNA was gel purified and ligated into pGEX-4T3 digested with the same enzymes. The resulting plasmid was designated pGEX-4T3-Ss $\lambda 20$. The expression vector pGEX-4T3-Ss $\lambda 20 \Delta B 3$ was generated by digestion of pGEX-4T3Ss $\lambda 20$ with BamHI and religation of the resulting vector after removal of $1156 \mathrm{bp}$ containing the 5 '-end of the Ss $\lambda 20$ cDNA clone. The nucleotide sequences of pGEX4T3-Ss $\lambda 20$ and pGEX-4T3-Ss $\lambda 20 \Delta$ B3 and their relevant open reading frames were confirmed by sequencing.

\subsection{Purification of recombinant proteins}

The recombinant proteins were produced in transformed BL21 E. coli cultures induced with $100 \mu \mathrm{M}$ isopropyl- $\beta$-D thiogalactopyranoside (IPTG) for $4 \mathrm{~h}$ and purified by affinity chromatography using a 
glutathione-Sepharose 4B column (Amersham Biosciences, Barcelona, Spain) according to the manufacturer's instructions. The sarcoptes-derived polypeptides were excised from the GST by thrombin cleavage and then analysed by SDS-PAGE, western blot and dot blot. The purified protein was quantified using the Bradford method [4] and then used to immunise rabbits or in ELISA tests.

\subsection{Western blotting}

After SDS-polyacrylamide (10\%) gel electrophoresis, the proteins were transferred onto $0.45 \mu \mathrm{m}$ pore size nitrocellulose membranes (Millipore, Billerica, MA, USA) using a Mini Protean II (Bio-Rad, Hercules, CA, USA) electroblotting apparatus at $100 \mathrm{~V}$ for $1 \mathrm{~h}$ in $25 \mathrm{mM}$ Tris/192 $\mathrm{mM}$ glycine buffer, $\mathrm{pH} 8.3$ containing $20 \%$ methanol. After a blocking step the specific antigens were revealed with a rabbit anti-Ss $\lambda 20 \Delta B 3$ polyclonal serum or a chamois infected serum followed by the addition of the appropriate speciesspecific peroxidase-conjugated secondary antibody. The immunocomplexes were revealed using 4-chloro-1-naftol as substrate. For dot blot analyses appropriate amounts of the purified Ss $\lambda 20 \Delta B 3$ polypeptide were spotted onto a nitrocellulose filter using a dot blot apparatus (BioRad) and processed as described for Western blot.

\subsection{Preparation of antisera}

One New Zealand White rabbit was immunised with the purified protein (Ss $\lambda 20 \Delta$ B3) for antibody production. The immunisation protocol consisted of 5 intramuscular injections at days 1, 8, 15, 28 and 56, using $200 \mu \mathrm{g}$ of the purified protein emulsified with complete Freund's adjuvant. The last boost was intravenous and without Freund's adjuvant. The serum from the bleed on day 28 and the preimmune serum from the same rabbit were used for immunological studies.

\subsection{ELISA test}

ELISA plates (96 well flat bottom plates, Corning) were coated overnight $(18 \mathrm{~h})$ at $4{ }^{\circ} \mathrm{C}$ with $100 \mathrm{ng}$ of purified Ss $\lambda 20 \Delta B 3$ diluted in $100 \mu \mathrm{L}$ of carbonatebicarbonate buffer (Sigma). The plates were then washed once with $200 \mu \mathrm{L}$ of PBS containing $0.05 \%$ Tween 20 (PBS-T) and blocked for $1 \mathrm{~h}$ at room temperature with $150 \mu \mathrm{L}$ of $1 \%$ yeast extract prepared in PBS-T. After washing once with PBS$\mathrm{T}, 100 \mu \mathrm{L}$ of each serum (diluted 20 times in blocking solution) were added to the appropriate wells and incubated for $1 \mathrm{~h}$ at $37^{\circ} \mathrm{C}$. The plates were then washed 5 times with $200 \mu \mathrm{L}$ of PBS-T and incubated with $100 \mu \mathrm{L}$ of the appropriate species-specific secondary antibody for $1 \mathrm{~h}$ at $37{ }^{\circ} \mathrm{C}$. ImmunePure peroxidase-conjugated Protein $G$ was used for chamois and deer sera (diluted 1500 times in blocking solution). After five washes, as described above, the plates were developed using o-phenylenediamine as substrate. The reaction was stopped with $100 \mu \mathrm{L}$ per well of $3 \mathrm{M}$ sulphuric acid. The absorbance at $450 \mathrm{~nm}$ was measured in a microplate reader (Sigma, model 680). For optimisation of antigen, sera and conjugate concentrations, two reference chamois sera were used. The positive serum (200/04) was obtained from an infected animal and the negative reference serum (129) was taken from a sarcoptes-free chamois. The results were expressed as a relative net optical density (relative $\mathrm{OD}_{450 \mathrm{~nm}}$ ) that was calculated according to the following formula:

$$
\mathrm{OD}=\frac{\text { ODunknown_serum-ODnegative_serum }}{\text { ODpositive_serum-ODnegative_serum }}
$$

The cut-off levels for the ELISA test were calculated as the mean of the 
relative $\mathrm{OD}_{450 \mathrm{~nm}}$ from scabies-free animals plus three times the standard deviation $[7,13,37]$.

\subsection{Immunolocalisation of $\operatorname{Ss} \lambda 20$ S. scabiei antigen}

Skin samples from a $S$. scabiei infected chamois were fixed in $10 \%$ neutral formalin and embedded in paraffin using standard procedures. For the immunohistochemical study, $4 \mu \mathrm{m}$ sections were immunostained using the peroxidaseantiperoxidase (PAP) method [35]. Briefly, the sections were cut, deparaffinised, rehydrated and rinsed with tap water. Afterwards, samples were treated to inactivate the endogenous peroxidase by incubation with methanol containing $3 \%$ $\mathrm{H}_{2} \mathrm{O}_{2}$ for $10 \mathrm{~min}$, washed with water for $10 \mathrm{~min}$ and then treated to prevent unspecific binding by a $15 \mathrm{~min}$ incubation with $10 \%$ normal swine serum (DAKO, Glostrup, Denmark), 3\% BSA in TBS (5 mM Tris/HCl pH 7.6, $136 \mathrm{mM}$ $\mathrm{NaCl}$ ). The tissue sections were incubated overnight at $4{ }^{\circ} \mathrm{C}$ with $\mathrm{Ss} \lambda 20 \Delta \mathrm{B} 3$ specific polyclonal rabbit-antiserum diluted 1:1000 in TBS and then washed three times with TBS. Then, the samples were incubated with swine anti-rabbit serum (DAKO) diluted 1:50 in TBS for $30 \mathrm{~min}$ at room temperature and washed three times with TBS followed by incubation with a rabbit PAP (soluble complexes of rabbit antibody to horseradish peroxidaseantihorseradish peroxidase) diluted 1:320 in TBS for $30 \mathrm{~min}$ at room temperature. Finally, the sections were incubated with the substrate 3, 3'-diaminobenzidine tetrahydrochloride (DAB, Sigma) for $10 \mathrm{~min}$ and washed with TBS and water. After staining with haematoxylin the preparations were dehydratated, pasted with DPX mountant for histology (Fluka) and observed using an Olympus BH-2 light microscope and photographed using a Olympus DP-12 digital camera.

\section{RESULTS}

\subsection{Isolation of cDNA clones coding for $S$. scabiei antigens}

In order to find a candidate molecule for the immunodiagnosis of S. scabiei we screened $1.0 \times 10^{6} \mathrm{PFU}$ from the $S$. scabiei var. hominis amplified library $\mathrm{Yv} 4$, with a serum sample from a naturally infected chamois. In this first screening, we selected 20 clones showing the strongest reactions (named Ss $\lambda 1$ to Ss $\lambda 20$ ). After additional rounds of screening only 11 out of the 20 clones initially selected remained strongly positive (Ss $\lambda 2$, 3, 4, 5, 6, 8, 9, 11, 12, 15, and 20). After in vivo excision of the $\mathrm{pBK}-\mathrm{CMV}$ phagemids from the positive $\lambda$ ZAP express vectors the presence of a cDNA insert was confirmed by EcoRI and $\mathrm{XhoI}$ digestion. To further characterise the cDNA $(0.7 \mathrm{~kb}$ to $2.0 \mathrm{~kb})$ in the recombinant $\mathrm{pBK}-\mathrm{CMV}$ phagemids, we investigated their nucleotide sequences using $\mathrm{T} 7$ and $\mathrm{T} 3$ primers. The resulting nucleotide sequences allowed their classification into three groups. Group one included clones Ss $\lambda 2$ and 11; group two clones Ss $\lambda 8$ and 12 and group three included the remaining 7 cDNA clones Ss $\lambda 3,4,5,6,9,15$ and 20 . We decided to focus our studies on Ss $\lambda 20$ cDNA because it contained the largest cDNA insert (2001 bp, excluding the polyA tail). In addition, considering the number of Ss $\lambda 20$-related positive clones identified, it possibly derived from an abundant parasite mRNA. Moreover, the strong antibody response against the positive clones might indicate that they encode an immunodominant antigen in infected animals. 


\subsection{Sequence analysis of the $S$. scabiei Ss $\lambda 20$ cDNA}

The nucleotide sequence of Ss $\lambda 20$ cDNA (Fig. 1A) was 2001 nt long and has been deposited in EMBL Nucleotide Sequence Database under the accession number AM286740. The first 1236 nucleotides included 21 well-conserved tandem repeats of 57 to $60 \mathrm{nt}$ residues. Most of the changes observed within this region occurred at the third codon position of the putatively encoded polypeptide and did not promote changes of the corresponding amino acid residues. From nucleotide 1237 to 1705 the repeated sequence was less conserved and from residue 1706 the sequence was unique showing no obvious repeats.

As expected from the cloning strategy used, the 5'-end of the parasite mRNA was not represented in the cDNA. The Ss $\lambda 20$ cDNA contained a 1930 bp open reading frame starting at the cDNA 5'-end and ending at a TGA codon located at nt 192830. This cDNA had a 3'-untranslated region of 71 residues preceding the poly(A) tail. A consensus polyadenylation signal was located 21 nucleotides upstream of the poly(A) tail. This ORF had the coding capacity for a 642 amino acid residue polypeptide with a calculated molecular mass of $60 \mathrm{kDa}$. As deduced from the cDNA sequence, the $\mathrm{N}$-terminal region of the $S$ s $\lambda 20$ polypeptide was made of 21 tandem repeats of a 19-20 amino acid sequence (Fig. 1B), followed by 156 residues whose repetition unit was less conserved and ending with 54 non-repeated residues preceding the stop codon. The residues 4 to 6 (AGA), 10 (S) and 13 to 17 (RYGQG) of the repeat unit were identical in all the repeats while the rest of the positions showed different degrees of variability. This protein region was very rich in the small amino acids Ala (12.7\%), Gly (25.3\%) and Ser $(25.7 \%)$. It should be mentioned that the carboxyl terminus unique sequence contained a high percent ( 21 out of the last 54 residues) of glutamate.

The pattern of amino acid repetition was best visualised (Fig. 1C) by plotting the hydropathicity index using 9 residues as window size. No transmembrane regions were predicted when using a 19 residue window (not shown).

No obvious match of the cDNA or deduced protein sequences could be identified in the databases, although some degree of homology was found with genes encoding other glycine-rich proteins.

\subsection{Production of $S$. scabiei recombinant proteins}

The full cDNA from the Ss $\lambda 20$ clone was inserted into the pGEX-4T3 expression vector as a fusion with GST resulting in expression vector $\mathrm{pGEX}-4 \mathrm{~T} 3-\mathrm{S} s \lambda 20$. The GST-Ss $\lambda 20$ fusion protein, with a calculated molecular mass of $95 \mathrm{kDa}$, was produced at very low levels in transformed BL21 E. coli cultures, and could not be purified by affinity chromatography in appropriate yields for further studies (data not shown).

In order to increase the level of recombinant protein expression and to take advantage of the presence of a suitable BamHI restriction site, we produced an $\mathrm{N}$-terminal deleted version of the Ss $\lambda 20$ polypeptide by digestion of the pGEX-4T3-Ss $\lambda 20$ vector with $B a m H I$ restriction endonuclease, resulting in the removal of $1156 \mathrm{bp}$ from the 5'-end of the clone maintaining the open reading frame of the fusion protein. The resulting plasmid pGEX-4T3Ss $\lambda 20 \Delta B 3$, encoded 263 residues from the carboxy terminus of the Ss $\lambda 20$ polypeptide fused to GST. The fusion protein GSTSs $\lambda 20 \Delta B 3$ had a predicted molecular mass of $53.0 \mathrm{kDa}$ and contained only one complete amino acid repeat (the 21 st repeat present in the $S s \lambda 20$ protein, see Fig. 1B) 
preceding the unique C-terminus. The GST-Ss $\lambda 20 \Delta B 3$ fusion protein was produced in transformed BL21 E. coli cultures and purified by affinity chromatography. The sarcoptes moiety, was cleaved from the GST using the protease thrombin and the purified protein was analysed by SDSPAGE (Fig. 2, panel A) and Western blot (Fig. 2, panel B).

The fusion protein GST-Ss $\lambda 20 \Delta \mathrm{B} 3$ showed an unexpected low electrophoretic mobility in SDS-PAGE indicating a molecular mass higher than the calculated mass for the recombinant protein (Fig. 2, panel A, lanes 1 and 2). Nevertheless, it was recognised by the positive serum from an infected chamois (Fig. 2, panel B, lanes 1 and 2). After thrombin cleavage, the purified truncated polypeptide Ss $\lambda 20 \Delta B 3$ also showed abnormal SDS-PAGE mobility indicating a molecular mass approximately twice the expected mass for the encoded polypeptide (28.9 kDa) (Fig. 2, panel A, lane 3). Furthermore, this polypeptide did not bind to nitrocellulose or PVDF filters after electrophoretic transfer (neither to $0.45 \mu \mathrm{m}$ nor to $0.2 \mu \mathrm{m}$ filters). Hence, the sarcoptes moiety could not be detected by Western blot (Fig. 2, panel B, lane 3). However, the purified polypeptide was recognised by the serum from an infected chamois when spotted onto a nitrocellulose filter using a dot blot apparatus (Fig. 2, panel C). The GST moiety that remained bound to the affinity column after thrombin cleavage was of the expected size (Fig. 2, panel A, lane 4) and indicated that the fusion protein was completely digested. As predicted, the GST was not recognised by the serum of the sarcoptesinfected chamois (Fig. 2, panel B, lane 4) indicating that the positive reactions observed (Fig. 2, panel B, lanes 1 and 2) were due to the $S s \lambda 20 \Delta B 3$ sequence.

The purified Ss $\lambda 20 \Delta B 3$ protein was used to produce a specific antiserum in a rabbit following the protocol described in the Materials and methods section.

\subsection{Identification and localisation of the native parasite $S s \lambda 20$ antigen}

Concentrated S. scabiei crude extracts were first analysed by SDS-PAGE and showed a complex protein pattern after silver staining (Fig. 3, panel A). Similar SDS-PAGE separated proteins were then transferred onto nitrocellulose membranes, which were then incubated with rabbit preimmune (Fig. 3 panel C) or the paired hyperimmune serum raised against the recombinant Ss $\lambda 20 \Delta \mathrm{B} 3$ polypeptide (Fig. 3 panel B). A broad reacting zone was observed between the 67 and $94 \mathrm{kDa}$ markers in the filters incubated with the hyperimmune serum (Fig. 3, panel B) while no reactions were observed when using the preimmune serum (Fig. 3, panel C). Considering that the calculated molecular mass for the Ss $\lambda 20$ polypeptide encoded in the Ss $\lambda 20$ cDNA was $60.0 \mathrm{kDa}$ and taking into account that the 5 '-end of the gene was not fully included in the cDNA it was expected that the corresponding S. scabiei native polypeptide had a molecular size larger than $60 \mathrm{kDa}$.

As expected, sera from mange-infected chamois recognised several faint protein bands in the mite crude extract (data not shown).

In order to locate the $S$ s $\lambda 20$ antigen in parasite organs or tissues, we carried out immunostaining of chamois skin sections that were highly infected with $S$. scabiei mites. Staining with the rabbit antiSs $\lambda 20 \Delta B 3$ polyclonal serum was specific to particular regions of the parasite (Fig. 4, panel B), while no staining was observed using preimmune serum (Fig. 4, panel A). More specifically, the integument of the epidermis and the spaces surrounding the parasite's vital organs were stained, while no host tissue staining was detected. The serum was also able to detect the antigen in skin sections derived from an infected fox (Vulpes vulpes) (see Fig. 4, panels C and D). 
$\begin{array}{llllllllllllllllllllll}S & S & G & A & V & A & G & A & G & S & S & S & K & G & R & Y & G & Q & G & S\end{array}$ CTCATCTGGCGCTGTAGCTGGGGCAGGATCATCTTCGAAAGGCCGATATGGACAAGGAAG $\begin{array}{lllllllllllllllllllllllll}S & S & G & A & L & A & G & A & G & S & T & S & N & G & R & Y & G & Q & G & S\end{array}$ CTCATCTGGTGCTTTGGCTGGAGCTGGATCCACTTCAAATGGCCGATATGGACAAGGAAG $\begin{array}{llllllllllllllllllll}S & S & T & \text { L } & A & G & A & G & S & S & S & N & S & R & Y & G & Q & G & S & S\end{array}$ CTCATCAACATTAGCTGGAGCTGGATCATCTTCAAATAGCCGATATGGACAAGGAAGCTC $\begin{array}{lllllllllllllllllllll} & F & G & A & V & A & G & A & G & S & S & S & K & G & K & Y & G & Q & G & S & S\end{array}$ ATTTGGCGCTGTAGCTGGCGCAGGATCCTCTTCGAAAGGCAAATATGGACAAGGAAGCTC $\begin{array}{llllllllllllllllllll}S & A & S & A & G & A & G & S & S & S & N & G & R & Y & G & Q & G & S & S & S\end{array}$ ATCTGCATCAGCTGGGGCTGGATCATCTTCAAATGGAAGATATGGACAAGGAAGCTCATC $\begin{array}{llllllllllllllllllll}A & S & A & G & A & G & A & S & S & N & G & R & Y & G & \mathcal{Q} & G & G & Y & S & G\end{array}$ TGCATCAGCTGGGGCTGGAGCCTCTTCAAATGGTAGATATGGACAAGGTGGCTATTCTGG

$\begin{array}{lllllllllllllllllllll}A & L & A & G & A & K & S & S & S & K & G & R & Y & G & Q & G & G & Y & S & G\end{array}$ TGCTCTAGCTGGCGCAAAATCATCTTCAAAAGGCAGATATGGACAGGGTGGTTATTCTGG $\begin{array}{llllllllllllllllllll}A & L & A & G & A & G & S & S & S & K & S & R & Y & G & Q & G & G & Y & S & G\end{array}$ TGCTCTAGCAGGCGCAGGATCATCTTCAAAAAGCAGATATGGACAAGGTGGTTATTCTGG $\begin{array}{llllllllllllllllllll}A & L & A & G & A & G & S & S & S & K & G & R & Y & G & Q & G & S & S & S & G\end{array}$ TGCTCTAGCAGGCGCAGGATCATCTTCAAAAGGTAGATATGGACAAGGAAGCTCATCTGG $\begin{array}{llllllllllllllllllll}A & L & A & G & A & G & S & S & S & N & G & R & Y & G & Q & G & S & S & S & A\end{array}$ TGCTTTGGCTGGTGCTGGATCCTCTTCAAATGGCAGATATGGACAAGGAAGCTCATCTGC $\begin{array}{llllllllllllllllllll}L & A & G & A & G & S & S & S & N & G & R & Y & G & Q & G & G & S & S & A & L\end{array}$ ATTAGCTGGGGCTGGATCATCTTCAAATGGTAGATATGGACAAGGAGGCTCATCTGCATT $\begin{array}{llllllllllllllllllll}A & G & A & G & S & S & S & Q & G & R & Y & G & Q & G & G & Y & S & G & A & L\end{array}$ AGCTGGGGCTGGATCATCTTCTCAAGGCAGATATGGACAAGGTGGCTATTCTGGTGCTCT

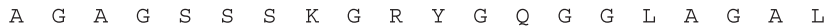
AGCTGGCGCAGGATCATCTTCAAAAGGCAGATATGGACAAGGAGGTTTAGCTGGTGCTTT

$\begin{array}{llllllllllllllllllllllllllll}A & G & A & G & S & S & S & N & G & R & Y & G & Q & G & S & S & S & G & A & L\end{array}$ GGCTGGTGCTGGATCCTCTTCGAATGGCAGATATGGACAAGGAAGCTCATCTGGTGCTTT

$\begin{array}{llllllllllllllllllll}A & G & A & G & S & S & S & N & G & R & Y & G & Q & G & S & S & S & A & L & A\end{array}$ GGCTGGTGCTGGATCCTCTTCAAATGGCAGATATGGACAAGGAAGCTCATCTGCATTAGC

$\begin{array}{llllllllllllllllllllllll}G & A & G & S & S & S & N & G & R & Y & G & Q & G & G & S & S & A & L & A & G\end{array}$ TGGGGCTGGATCATCTTCAAATGGTAGATATGGACAAGGAGGCTCATCTGCATTAGCTGG

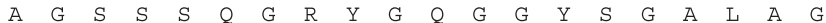
GGCTGGATCATCTTCTCAAGGCAGATATGGACAAGGTGGCTATTCTGGTGCTCTAGCTGG $\begin{array}{llllllllllllllllllllll}A & G & S & S & S & K & G & R & Y & G & Q & G & S & S & T & A & S & A & G & A\end{array}$ CGCAGGATCATCTTCAAAAGGCAGATATGGACAAGGAAGCTCAACTGCATCAGCTGGGGC $\begin{array}{lllllllllllllllllllll}G & S & S & S & N & G & R & Y & G & Q & G & G & S & S & A & L & A & G & A & G\end{array}$ TGGATCATCTTCAAATGGAAGATATGGACAAGGAGGCTCATCTGCTTTAGCTGGGGCTGG $\begin{array}{lllllllllllllllllllllllllll}S & T & S & N & G & R & Y & G & Q & G & S & S & S & A & L & A & G & A & G & S\end{array}$ ATCCACTTCAAATGGCCGATATGGACAAGGAAGCTCATCAGCATTAGCTGGAGCTGGATC $\begin{array}{llllllllllllllllllll}S & S & Q & G & R & Y & G & Q & G & S & Y & S & G & S & L & A & E & A & G & T\end{array}$ ATCTTCACAAGGCAGATATGGACAAGGAAGCTATTCTGGTTCTTTGGCTGAAGCTGGAAC $\begin{array}{llllllllllllllllllll}G & S & S & S & Y & S & I & D & E & D & V & S & L & S & G & E & S & S & Y & Y\end{array}$ TGGATCATCTTCATATAGTATAGATGAAGATGTTAGCTTATCTGGTGAATCATCTTACTA

$\begin{array}{lllllllllllllllllllll}D & E & S & G & Q & G & S & S & S & G & T & L & D & G & A & E & S & S & Y & Y\end{array}$ TGATGAATCTGGACAAGGAAGTTCCTCTGGCACTTTGGATGGGGCTGAATCATCTTACTA $\begin{array}{llllllllllllllllllllllll}D & G & F & E & O & E & S & Y & S & G & A & L & G & E & A & E & S & S & N & N\end{array}$ TGATGGATTTGAACAAGAAAGCTATTCTGGCGCTTTGGGTGAAGCTGAATCATCTAACAA $\begin{array}{lllllllllllllllllllll}D & G & T & E & Q & G & S & Y & S & G & A & L & A & G & A & G & S & S & Y & Y\end{array}$ TGATGGAACTGAACAAGGAAGCTATTCTGGCGCTTTAGCTGGAGCTGGATCTTCTTACTA

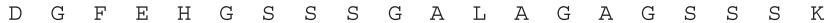
TGATGGATTTGAACACGGAAGTTCATCTGGTGCTTTAGCTGGAGCTGGATCATCTTCGAA $\begin{array}{llllllllllllllllllll}G & G & H & G & S & G & S & S & S & A & L & A & G & S & G & S & S & S & D & S\end{array}$ AGGTGGACATGGTAGCGGAAGTTCATCTGCATTAGCTGGATCTGGATCTTCTTCAGATAG

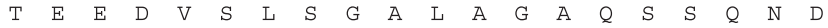
TACAGAAGAAGATGTTAGCTTATCTGGTGCTTTAGCAGGAGCTCAATCTTCACAAAATGA $\begin{array}{llllllllllllllllllll}G & Y & E & Q & G & S & L & S & G & S & L & A & G & T & G & S & S & S & N & G\end{array}$ TGGGTATGAACAAGGAAGCTTATCTGGTTCTTTGGCTGGCACTGGATCATCTTCGAATGG $\begin{array}{lllllllllllllllllllll}N & H & G & Y & G & G & S & T & D & S & S & E & S & S & S & E & E & F & E & E\end{array}$ AAATCATGGTTACGGAGGTTCCACTGATTCTTCTGAATCAAGTTCTGAAGAGTTTGAAGA $\begin{array}{llllllllllllllllllll}I & I & E & E & E & E & V & E & E & M & V & E & E & E & V & E & E & E & F & V\end{array}$ AATTATCGAAGAAGAAGAGGTTGAAGAAATGGTCGAAGAAGAAGTTGAAGAAGAATTTGT

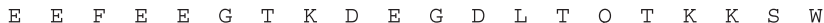
AGAGGAGTTTGAAGAAGGAACAAAAGATGAAGGAGATCTAACTCAAACGAAAAAAAGCTG $\mathrm{K} \mathrm{K}$ *

GAAGAAATGAATAAATTTGTTTAATTTTATGAAAAATTTTTGGAATTTCTCAAAAATAAA ATGAAAAAGATTATAATTTCGAAAAAAAAAAAAAAAAAA 


\section{B}

$\begin{array}{lllll}1 & 5 & 10 & 15 & 20\end{array}$

Cons : GALAGAGSSSNGRYGQGSSS
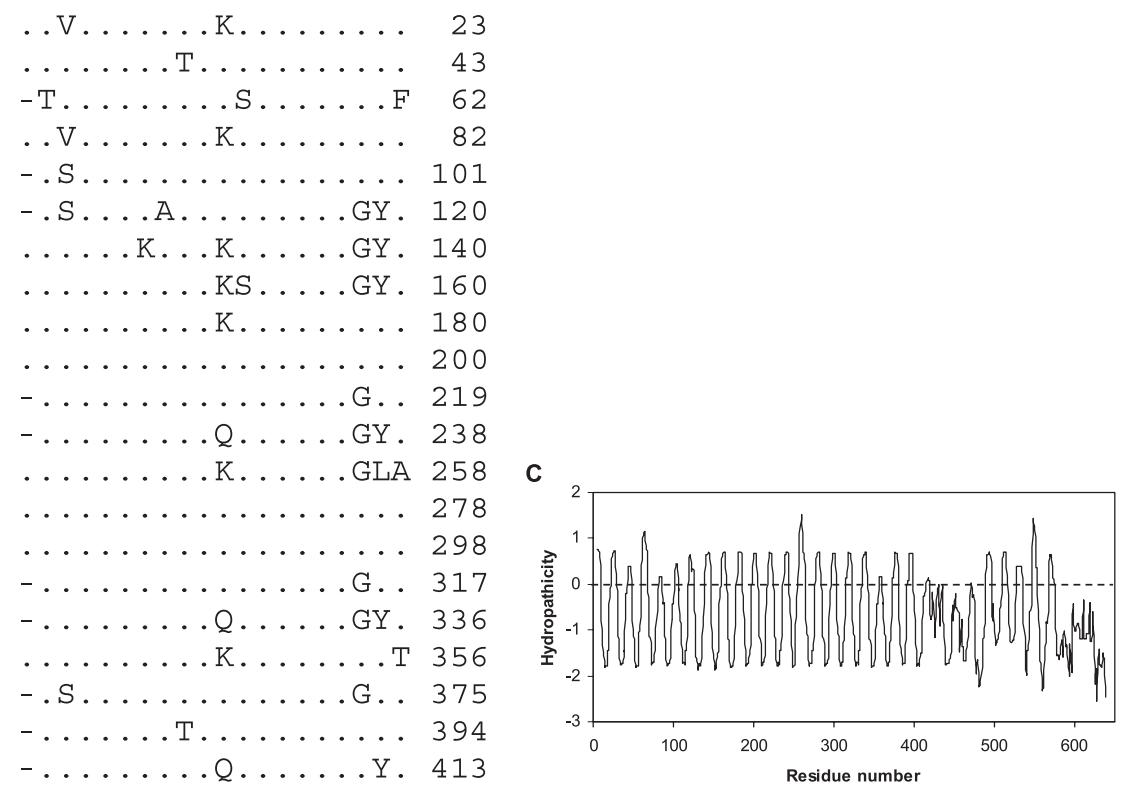

Figure 1. (A) Nucleotide sequence of Sarcoptes scabiei Ss $\lambda 20$ cDNA and amino acid sequence of the predicted translation product. The BamHI restriction site used for the construction of pGEX$4 \mathrm{~T} 3-\mathrm{S} s \lambda 20 \Delta \mathrm{B} 3$ is underlined. The polyadenylation signal is double underlined. The numbers on the right vertical axis refer to the amino acid position in the Ss $\lambda 20$ cDNA. (B) Alignment of the tandem repeated sequences derived from Ss $\lambda 20 \mathrm{cDNA}$. The numbers on the right vertical axis correspond to amino acid residue numbers in the Ss $\lambda 20$ deduced polypeptide. The dots indicate identity with respect to the consensus sequence (cons) and the dashes correspond to sequence gaps. (C) Hydropathicity profile of the Ss $\lambda 20$ polypeptide.

\subsection{Development of an ELISA test for the diagnosis of scabies}

One of the aims of this study was to develop an ELISA test to detect antibodies to $S$. scabiei in the sera of infected animals. Therefore, the potential of the recombinant antigen $S s \lambda 20 \Delta B 3$ as an immunodiagnostic molecule was assessed. The ELISA method was validated with a panel of 76 sera samples: 40 from chamois and 36 from deer, of which 10 were obtained from scabietic animals (mites were isolated from skin lesions present in the dead animals) and 66 were from healthy unexposed individuals originating from a scabies-free area. The ELISA tests were performed as described in the Materials and methods section. Positive and negative reference sera for each animal species were included on each ELISA plate.

The results obtained for the 76 analysed sera are shown in Figure 5. The cut-off levels for the ELISA test were 0.43 for deer and 0.16 for chamois sera. These cut-off values were also used to calculate the sensitivity and specificity of the test. Thus, the ELISA test using the Ss $\lambda 20 \Delta \mathrm{B} 3$ 
A

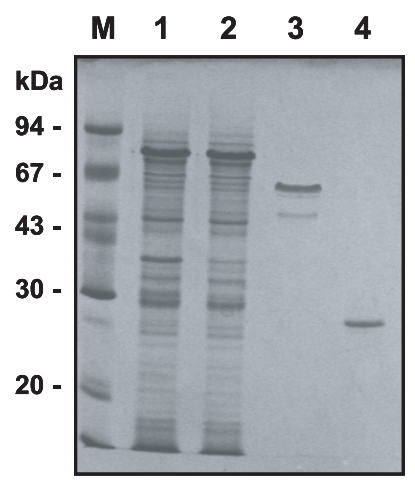

B

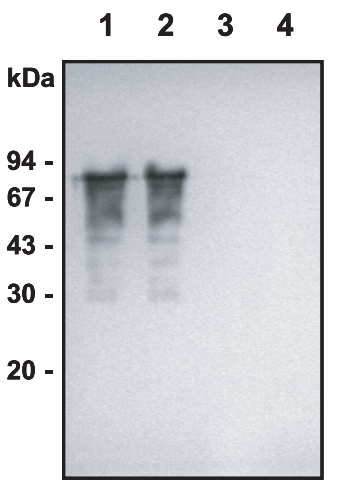

C

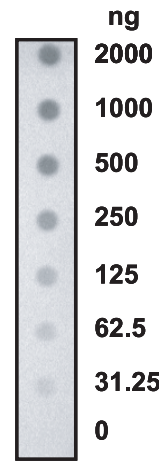

Figure 2. Analysis of Ss $\lambda 20 \Delta \mathrm{B} 3$ expression and purification. (A) Coomassie Blue stained SDSPAGE gel. Lane 1, crude cell extract from pGEX-Ss $\lambda 20 \Delta \mathrm{B} 3$-transformed, IPTG-induced $E$. coli; lane 2, cell-free extract; lane 3, purified Ss $\lambda 20 \Delta \mathrm{B} 3$ sarcoptes moiety; lane 4, GST bound to the affinity column after cleavage with thrombin; M, low molecular weight protein markers (Amersham Biosciences). (B) Western blot analysis of a similar SDS-PAGE gel using a serum sample from a sarcoptes-infected animal. (C) Dot blot analysis of the purified $\mathrm{S} s \lambda 20 \Delta \mathrm{B} 3$ protein.
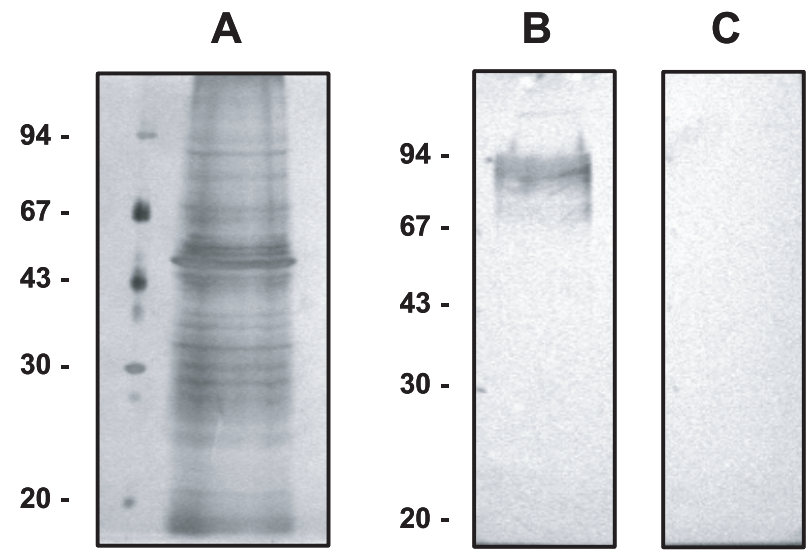

Figure 3. SDS-PAGE and Western blot analysis of crude extracts from Sarcoptes scabiei. (A) Silverstained gel. (B) Immunodetection using a hyperimmune serum raised against purified recombinant Ss $\lambda 20 \Delta \mathrm{B} 3$ polypeptide. (C) Immunodetection using a paired preimmune serum. The numbers on the left margin indicate molecular weight markers $(\mathrm{kDa})$.

recombinant antigen was characterised by $100 \%$ sensitivity and $97 \%$ specificity in both animal species. The $97 \%$ specificity value was due to the presence of antibodies (above the cut-off level) against $S$. scabiei in one out of the 33 deer and one out of 33 chamois from scabies-free areas. It should be mentioned that in the case of the chamois the only "false positive animal" gave a relative $\mathrm{OD}_{450 \mathrm{~nm}}$ of 0.201 , which is very close to the cut-off established for the test. Given that all of the relative $\mathrm{OD}_{450 \mathrm{~nm}}$ for the positive animals are well above 0.16 we could also consider a practical cut-off 

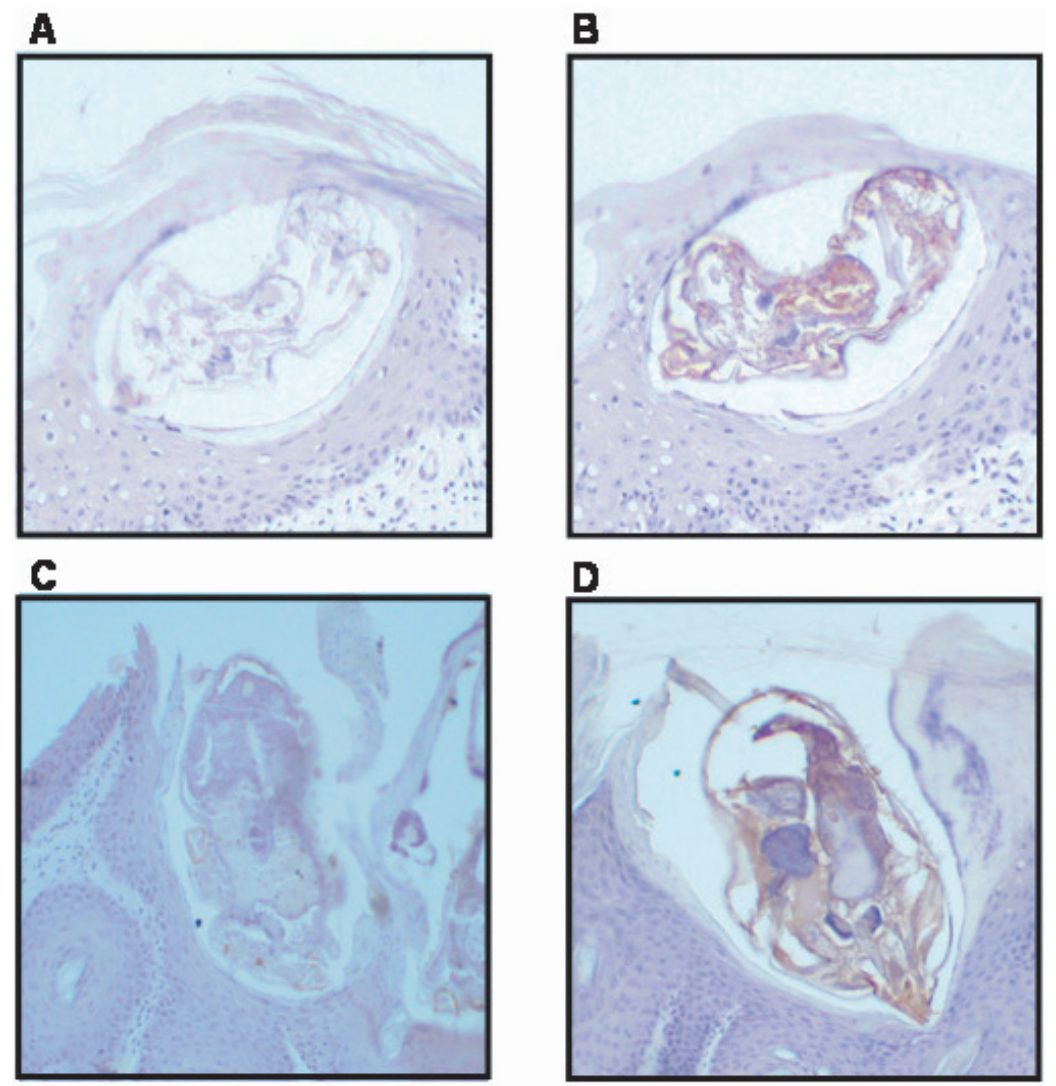

Figure 4. Immunostaining of adult Sarcoptes scabiei Ss $\lambda 20$ antigen in skin sections derived from a mange-infected chamois (panels A, B) or a mange-infected fox (panels C, D). Peroxidase antiperoxidase immunostaining after reaction with a preimmune serum $(\mathrm{A}, \mathrm{C})$, or a rabbit polyclonal anti-Ss $\lambda 20 \Delta B 3$ serum (B, D). Sera samples were used at 1:1000 dilution. Image magnification $(\times 100)$.

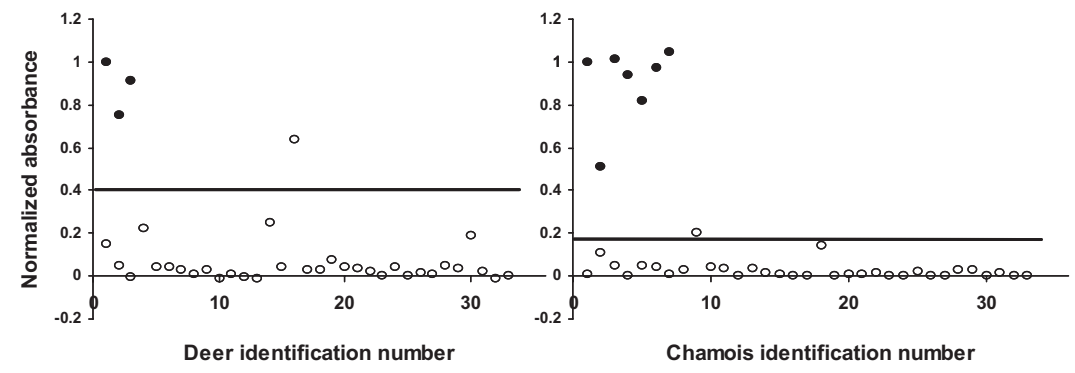

Figure 5. ELISA test for the detection of antibodies against recombinant Ss $\lambda 20 \Delta B 3$ protein in sera samples from free-ranging deer or chamois. Filled and open circles represent sera from infected or healthy animals, respectively. The cut-off for each animal species is represented by a horizontal line. 
of 0.25 which would increase the specificity of the test to $100 \%$.

The diagnostic value of a test also depends on the degree of cross-reaction with antigens from other related parasites. Given the lack of sequence similarity with respect to other antigens in the databases and considering that in Asturias (in the northwest of Spain) the animals diagnosed with sarcoptic mange are often also infected with ticks (Arthropod, Arachnida, Ixodes ricinus) we checked whether the sera obtained from animals infected with ticks had cross-reactivity with the $S$. scabiei recombinant antigen. The ELISA test described in this work did not show positive reactions with sera from one fallow deer (Dama dama) or five deer all diagnosed as tick-infected and sarcoptes-free. The $\mathrm{OD}_{450 \mathrm{~nm}}$ of these studied samples was in the range of the negative reference sera (data not shown).

\section{DISCUSSION}

We isolated by immunoscreening of a $S$. scabiei var. hominis library [10] a cDNA (Ss $\lambda 20)$ which was related to a group of six other cDNA whose nucleotide sequences are slightly different (these sequences will be reported elsewhere). The changes observed could be attributed to alternative splicing of the same precursor mRNA but further experiments would be necessary to demonstrate this hypothesis. Moreover, alternative polyadenylation sites have also been observed in these cDNA. In Ss $\lambda 20$, the polyadenylation signal was located 21 nucleotides upstream of the poly(A) tail while in $\mathrm{Ss} \lambda 5$ the polyadenylation signal was located 60 nucleotides before the poly(A) tail.

The polypeptide encoded by $\operatorname{Ss} \lambda 20$ cDNA was characterised by an N-terminus region showing tandem repetitions of a 19 to 20 residue glycine-serine rich sequence. The sequence of the repeated peptide had some homology with other proteins in the databases which also contain high levels of glycine and serine, such as fibroin, keratin or the spider silk protein and spidroin [8]. This sequence similarity was lost at the C-terminus region, where the amino acid sequence of the protein did not contain amino acid repeats and was not rich in glycine and serine.

It should be mentioned that two other expressed sequence tags (EST) with internal repeats (accession numbers BM276606 and BM276623) have been previously isolated from the same library [10]. These two EST had some homology with a glycinerich protein from the carrot (accession number T14306); they were probably fragments of the same cDNA considering their very high sequence homology. The Ss $\lambda 20$ cDNA reported in this work also encoded a glycine-rich protein $(25.3 \%)$, but did not have significant homology with respect to these two EST. Antigens bearing repetitive sequences have also been described in eukaryotic cells, including protozoan parasites $[14,17,22]$, as is the case of antifreeze proteins [34], collagens [26], apolipoprotein [21] and the mucins [11]. In some cases, the biological role of repeating sequences is known to be related to their particular three-dimensional structure. In the malaria circumsporozoite antigen [27] they have been related to the high antigenicity of this protein.

The antiserum raised against the recombinant antigen recognised, in a crude extract of S. scabiei, several protein bands ranging from 67 to $94 \mathrm{kDa}$. This result indicates that the isolated cDNA clone, with a coding capacity for a $60 \mathrm{kDa}$ polypeptide, did not include the full coding sequence. The protein bands recognised by the serum could represent different glycosylation patterns of the protein or they could be due to some degree of proteolysis or degradation in the mite extract. However, no consensus $\mathrm{N}$-glycosylation sequences (Asn-X-Ser or Asn-X-Thr) were 
found in the deduced polypeptide sequence. Taking into account the hypothesis that the different cDNA included in the $\mathrm{Ss} \lambda 20$ group could have originated by differential splicing, the heterogeneous size of the antigen could also be explained by the presence of a set of reactive proteins containing a variable number of amino acid repeats. The fusion protein GST-Ss $\lambda 20 \Delta \mathrm{B} 3$ as well as the purified truncated polypeptide Ss $\lambda 20 \Delta B 3$ showed an abnormal electrophoretic mobility in SDS-PAGE indicating a molecular mass higher than the calculated mass for the recombinant protein (Fig. 2, panel A). This anomalous migration has been previously described for proteins that have an uneven amino acid distribution [28]. In agreement with this, the Ss $\lambda 20 \Delta \mathrm{B} 3$ encoded protein has an unusual charge and amino acid asymmetry. The amino-terminal region containing the repeats is very rich in the small amino acids Ala (12.7\%), Gly $(25.3 \%)$ and Ser $(25.7 \%)$ while the carboxyl terminus unique sequence is more acidic containing a high percentage $(38 \%)$ of glutamate. The distribution of Ss $\lambda 20$ antigen in the parasite organs, the integument of the epidermis and the spaces surrounding the parasite's vital organs, seems to be similar to that of $S$. scabiei delta-class glutathione S-transferase [30].

Recently, an amplified capture enzymelinked immunosorbent assay was developed to detect specific antibodies to $S$. scabiei in chamois (Rupicapra spp.) serum [31]. This assay, the first one designed for the detection of $S$. scabiei in a freeranging ruminant host, used a whole body extract of the various developmental stages of S. scabiei as antigen, obtained from skin lesions of naturally infected red foxes (Vulpes vulpes). Although this test has been proven to be effective for the diagnosis of sarcoptic mange in wild ungulates (sensitivity 93\%, specificity 97\%), its main drawback is the requirement of using mites for antigen preparation. In addition, the existence of cross-reactions should also be expected, considering the complex nature of the antigen. In order to overcome these problems, we developed an ELISA test, based on the use of a $S$. scabiei specific recombinant antigen produced in $E$. coli, characterised by a very high sensitivity $(100 \%)$ and specificity $(97 \%)$. These values were calculated using the cut-off obtained from the analysis of a low number of samples (76 wild animals, 10 known positive and 66 negative samples) and it would certainly be desirable to use a larger number of sera in order to get higher confidence, sensitivity and specificity values. Nevertheless, it should be considered that wild fauna sample collection is very difficult and, in addition, identification of mite-infected free-ranging animals is a hard task. For these reasons, the relatively low number of samples used in this study were taken from dead animals that were found in the study areas or those killed by government-controlled shooting parties.

A unitary recombinant antigen has several advantages when compared to the use of a whole body extract of mites obtained from naturally infected animals: (1) the recombinant antigen can be reproducibly and safely produced in large quantities, (2) the production and purification methods are cheap and well established, and (3) it is less prone to cross-reactivity. It has been described that some mite body extracts are contaminated with host immunoglobulins that are ingested by the mite during infection [35]. Therefore, to avoid non-specific background reactions in the ELISA, these immunoglobulins must be eliminated, the process of which can lead to loss of antigen; it is also both expensive and time consuming.

The ELISA developed in this study was able to detect (with $100 \%$ efficacy) sick animals that showed sarcoptes macroscopic lesions, nevertheless we do not know its minimum level of detection or if 
the ELISA will be able to detect carrier animals with microscopic, invisible lesions. This remains a possibility given that the ELISA was very sensitive, and had very low background levels and cross-reactivity with other parasites.

It should also be mentioned that Sarcoptes mites from different host species are morphologically indistinguishable and $S$. scabiei is generally recognised as a unique species with different physiological varieties, adapted to groups of zoologically related host species. In this context, the validity of a test should also be extended to the analysis of sera from the various species putatively affected by this parasite.

Further studies involving experimental infections will be performed in order to determine the onset of the immune response against the $S s \lambda 20$. A recent study has shown that it is now possible to test cloned scabies antigens for vaccine potential [12] so we also plan to investigate the use of Ss $\lambda 20$ as a vaccine candidate.

\section{ACKNOWLEDGEMENTS}

We are very grateful to Dr D.J. Kemp (Malaria and Scabies Laboratory, Queensland Institute of Medical Research, Brisbane, Australia), for sending the Sarcoptes scabiei cDNA library, and to Dr Kevin Dalton for proofreading the text. R. Casais and J.M. Martín Alonso are recipients of a "Ramón y Cajal" contract from the Spanish Ministerio de Educación y Ciencia cofinanced by Fondo Social Europeo.

\section{REFERENCES}

[1] Arlian L.G., Runyan R.A., Sorlie L.B., Vyszenski-Moher D.L., Estes S.A., Characterization of Sarcoptes scabiei var. cannis (Acari: Sarcoptidae) antigens and induced antibodies in rabbits, J. Med. Entomol. (1985) 22:321-323.

[2] Arlian L.G., Vyszenski-Moher D.L., Gilmore A.M., Cross-antigenicity between Sarcoptes scabiei and the house dust mite, Dermatophagoides farinae (Acari: Sarcoptidae and Pyroglyphidae), J. Med. Entomol. (1988) 25:240-247.

[3] Arlian L.G., Vyszenski-Moher D.L., Ahmed S.G., Estes S.A., Cross-antigenicity between the scabies mite, Sarcoptes scabiei and the house dust mite, Dermatophagoides pteronyssinus, J. Invest. Dermatol. (1991) 96:349-354.

[4] Bradford M.M., A rapid and sensitive method for the quantitation of microgram quantities of protein utilizing the principle of protein-dye binding, Anal. Biochem. (1976) 72:248-254.

[5] Bornstein S., Zakrisson G., Humoral antibody response to experimental Sarcoptes scabiei var. vulpes infection in the dog, Vet. Dermatol. (1993) 4:107-110.

[6] Bornstein S., Thebo P., Zakrisson G., Evaluation of an enzyme-linked immunosorbent assay (ELISA) for the serological diagnosis of canine sarcoptic mange, Vet. Dermatol. (1996) 7:21-28

[7] Bornstein S., Wallgren P., Serodiagnosis of sarcoptic mange in pigs, Vet. Rec. (1997) 141:8-12.

[8] Colgin M.A., Lewis R.V., Spider minor ampullate silk proteins contain new repetitive sequences and highly conserved non-silklike "spacer regions", Protein Sci. (1998) 7:667-672.

[9] Fernández-Morán J., Gómez S., Ballesteros F., Quirós P., Benito J.L., Feliu C., Nieto J.M., Epizootiology of sarcoptic mange in a population of Cantabrian chamois (Rupicapra pyrenaica parva) in Northwest Spain, Vet. Parasitol. (1997) 73:163-171.

[10] Fischer K., Holt D.C., Harumal P., Curried B.J., Walton S.F., Kemp D.J., Generation and characterization of cDNA clones from Sarcoptes scabiei var. hominis for an expressed sequence tag library: identification of homologues of house dust mite allergens, Am. J. Trop. Med. Hyg. (2003) 68:61-64.

[11] Gendler S.J., Lancaster C.A., TaylorPapadimitriou J., Duhig T., Peat N., Burchell J., Pemberton L., Lalani N., Wilson D., Molecular cloning and expression of human tumor-associated polymorphic epithelial mucin, J. Biol. Chem. (1990) 265:1528615293.

[12] Hamural P., Morgan M., Walton S.F., Holt D.C., Rode J., Arlian L.G., Currie B.J., Kemp D.J., Identification of a homologue of a house dust mite allergen in a cDNA library from Sarcoptes scabiei var. hominis 
and evaluation of its vaccine potential in a rabbit/S. scabiei var. canis model, Am. J. Trop. Med. Hyg. (2003) 68:54-60.

[13] Hollanders W., Vercruysse J., Raes S., Bornstein S., Evaluation of an enzymelinked immunosorbent assay (ELISA) for the serological diagnosis of sarcoptic mange in swine, Vet. Parasitol. (1997) 69:117-123.

[14] Kemp D.J., Coppel R.L., Anders R.F., Repetitive proteins and genes of malaria, Annu. Rev. Microbiol. (1987) 41:181-208.

[15] Kimbita E.N., Xuan X., Huang X., Miyazawa T., Fukumoto S., Mishima M., Suzuki H., Sugimoto C., Nagasawa H., Fujisaki K., Suzuki N., Mikami T., Igarashi I., Serodiagnosis of Toxoplasma gondii infection in cats by enzyme-linked immunosorbent assay using recombinant SAG1, Vet. Parasitol. (2001) 102:35-44.

[16] Kyte J., Doolittle R.F., A simple method for displaying the hydropathic character of a protein, J. Mol. Biol. (1982) 157:105-132.

[17] Lafaille J.J., Linss J., Krieger M.A., SourtoPadrón T., de Souza W., Gooldenberg S., Structure and expression of two Trypanosoma cruzi genes encoding antigenic proteins bearing repetitive epitopes, Mol. Biochem. Parasitol. (1989) 35:127-136.

[18] Marín M.S., Prieto M., Martín J.M., Casais R., Boga J.A., Parra F., Identification and expression of a Fasciola hepatica gene encoding a gut antigen protein bearing repetitive sequences, Mol. Biochem. Parasitol. (1992) 55:155-165.

[19] Matthes H.F., Nockler K., Hiepe T., Klinischer Verlauf spontaner und experimenteller Sarcoptes suis-Infektionen beim Schwein, Monatsh. Veterinärmed. (1990) 45:706-709.

[20] Mattsson J.G., Ljunggren E.L., Bergstrom K., Paramyosin from the parasitic mite Sarcoptes scabiei: cDNA cloning and heterologous expression, Parasitology (2001) 122:555-562.

[21] McLean J.W., Tomliso J.E., Kuang W.J., Eaton D.L., Chen E.Y., Fless G.M., Scanu A.M., Lawn R.M., cDNA sequences of human apolipoprotein (a) is homologous to plasminogen, Nature (1987) 300:132-137.

[22] McMaster W.R., Button L.L., Wallis A.E., Frommel T., Webb J., Fujikura Y., Reiner N.E., Molecular genetics of repetitive antigens and the major surface glycoprotein of Leishmania, in: Agabian N., Cerami A. (Eds.), Parasites: Molecular biology, drug and vaccine design, Wiley-Liss, New York, 1990, pp. 183-190.
[23] Morgan M.S., Arlian L.G., Estes S.A., Skin test and radioallergosorbent test characteristics of scabietic patients, Am. J. Trop. Med. Hyg. (1997) 57:190-196.

[24] Morris D.O., Dunstan R.W., A histomorphological study of sarcoptic acariasis in the dog: 19 cases, J. Am. Anim. Hosp. Assoc. (1996) 32:119-124.

[25] Mount D.M., Conrad B., Improved programs for DNA and protein sequence analysis on the IBM personal computer and other standard computer systems, Nucleic Acids Res. (1986) 14:443-454.

[26] Myers J.C., Howard P.S., Jelen A.M., Dion A.S., Macarack E.J., Duplication of type IV collagen $\mathrm{COOH}$-terminal repeats and species-specific expression of $\alpha 1$ (IV) and $\alpha 2$ (IV) collagen genes, J. Biol. Chem. (1987) 262:9231-9238.

[27] Nussenzweig V., Nussenzweig R.S., Circumsporozoite proteins of malaria parasites, Cell (1985) 42:401-403.

[28] Ossorio P.N., Schwartzman J.D., Boothroyd J.C., A Toxoplasma gondii rhoptry protein associated with host cell penetration has unusual charge asymmetry, Mol. Biochem. Parasitol. (1992) 50:1-15.

[29] Pearson W.R., Lipman D.J., Improved tools for biological sequence comparison, Proc. Natl. Acad. Sci. USA (1988) 85:2444-2448.

[30] Pettersson E.U., Ljunggren E.L., Morrison D.A., Mattsson J.G., Functional analysis and localisation of a delta-class glutathione Stransferase from Sarcoptes scabiei, Int. J. Parasitol. (2005) 35:39-48.

[31] Rambozzi L., Menzano A., Lavin S., Rossi L., Biotin-avidin amplified ELISA for detection of antibodies to Sarcoptes scabiei in chamois (Rupicapra spp.), Vet. Res. (2004) 35:701-708.

[32] Rossi L., Meneguz P.G., DeMartin P., Rodolfi M., The epizootiology of sarcoptic mange in chamois from the Italian Eastern Alps, Parassitologia (1995) 37:233-240.

[33] Sambrook J., Fritsch E.F., Maniatis T., Molecular Cloning, A laboratory manual, 2nd edition, Cold Spring Harbor Laboratory Press, Cold Spring Harbor, NY, 1989.

[34] Scott G.K., Hew C.L., Davies P.L., Antifreeze protein genes are tandemly 
linked and clustered in the genome of the winter flounder, Proc. Natl. Acad. Sci. USA (1985) 82:2613-2617.

[35] Sternberger L.A., Hardy P.H., Cuculis J.J., Meyer H.G., The unlabeled antibody enzyme method of immunohistochemistry: preparation and properties of soluble antigenantibody complex (horseradish peroxidaseantihorseradish peroxidase) and its use in identification of spirochetes, J. Histochem. Cytochem. (1970) 18:315-333.
[36] Van der Heijden H.M.J.F., Rambags P.G.M., Elbers A.R.W., van Maanen C., Hunneman W.A., Validation of ELISAs for the detection of antibodies to Sarcoptes scabiei in pigs, Vet. Parasitol. (2000) 89:95-107.

[37] Zimmermann W., Kircher P., Serologische Bestandesuntersuchung und Sanierungsüberwachung der Sarcoptes scabiei var. suis Infektion: erste vorläufige Resultate, Schweiz. Arch. Tierheilk. (1998) 140:513517. 